\title{
VLADIMIROV-TYPE APPROXIMATION OF WIENER INTEGRALS OF SUITABLY DOMINATED FUNCTIONALS
}

\author{
BY \\ HENRY C. FINLAYSON
}

Introduction. Cameron [1] has given an approximation technique for the Wiener integral (over the space $C$ of real continuous functions $x(t)$ on $[0,1]$ such that $x(0)=0)$ of any functional from a class of smooth functionals dominated by a functional $H\left(\int_{0}^{1} w(t) x^{2}(t) d t\right)$ where $w(t) \equiv 1$ and $H$ is such that $H(u)$ is monotonically increasing on $[0, \infty]$ and, for an appropriate constant $\rho$, $H\left(\rho+2 \int_{0}^{1} w(t) x^{2}(t) d t\right)$ is Wiener integrable. In this paper the functionals from such a class, with more general $w(t)$ to be described below, will be dealt with. Vladimirov [6] has given yet another means of approximation for the Wiener integrals of functionals dominated in this way, still with the restriction $w(t) \equiv 1$. Vladimirov's approximation, which improves by increasing $n$, is given by a formula

$$
\int_{c} F[x(\cdot)] d x \doteq \sum_{k=-\infty}^{\infty} v_{k} R_{n, k}
$$

In this formula the $\nu_{k}$ 's are constants and the $R_{n, k}$ 's are $n$-fold Riemann integrals dependent on $F$ and also on a complete orthonormal set of functions of bounded variation. This latter set of functions is in turn dependent on $w(t)$ and for $w(t) \equiv 1$ turns out to be the odd harmonic cosine functions. It is the purpose of this paper to generalize Vladimirov's result by generalizing $w(t)$. $\S 1$ contains notation and the theorems' statements while $\$ 2$ and $\$ 3$ contain respectively nine lemmas and their proofs and the theorems' proofs.

1. Notation and statements of the theorems. Let $w(t)>0$ have a continuous third derivative on $[0,1]$. Let $\left\{\lambda_{n}\right\}$ and $\left\{\alpha_{n}(t)\right\}\left({ }^{1}\right): n=1,2,3, \ldots$ be the characteristic numbers and normalized characteristic functions of the Sturm-Liouville problem

$$
\left\{[1 / w(t)] \alpha^{\prime}(t)\right\}^{\prime}+\lambda \alpha(t)=0, \quad \alpha^{\prime}(0)=\alpha(1)=0 \quad \text { on }[0,1]
$$

Received by the editors December 19, 1966.

( $\left.{ }^{1}\right)$ The $B$ 's and $\alpha$ 's of Vladimirov's paper (in which $w(t) \equiv 1$ is used) are respectively the $\alpha$ 's and the normalized $\beta$ 's of this paper. 
The following notation will be used for $n=1,2,3, \ldots$ :

$$
\begin{aligned}
\beta_{n}(t) & =\int_{0}^{t} \alpha_{n}(s) d s \quad \text { for } t \in[0,1], \\
\gamma_{n} & =\int_{0}^{1} \beta_{n}^{2}(s) d s, \\
c_{n} & =\int_{0}^{1} \alpha_{n}(s) d x(s), \\
\sigma_{n} & =\left\{\gamma_{n}\left[\lambda_{n} \int_{0}^{1} s w(s) d s / 2+1\right]\right\}^{1 / 2}, \\
\nu_{n} & =\nu_{-n}=\gamma_{n} /\left[4 \sigma_{n}^{2}\right], \\
\nu_{0} & =1-2 \sum_{n=1}^{\infty} \nu_{n}
\end{aligned}
$$

(that $\sum_{n=1}^{\infty} 1 / \lambda_{n}$ converges, and thus $\nu_{0}$ is defined follows from Lemma 2.6),

$$
\begin{aligned}
x_{n}(t) & =-x_{-n}(t)=\sigma_{n} \beta_{n}(t) / \gamma_{n}^{1 / 2}, \\
x_{0}(t) & \equiv 0, \\
x^{n}(t) & =\sum_{i=1}^{n} c_{i} \beta_{i}(t), \\
\psi_{n}(\xi, t) & =\sum_{i=1}^{n} \xi_{i} \beta_{i}(t), \\
e_{n}(\xi) & =\pi^{-n / 2} \exp \left(-\xi_{1}^{2}-\cdots-\xi_{n}^{2}\right) .
\end{aligned}
$$

Whereas Vladimirov's paper concerns itself only with $w(t) \equiv 1$ the present paper deals with the more general case.

The two theorems of the paper will now be stated.

THEOREM 1. Let $F[x(\cdot)]$ be of either of the forms

$$
K_{0}+\sum_{i=1}^{3} \int_{0}^{1}(i) \int_{0}^{1} x\left(t_{1}\right) \cdots x\left(t_{i}\right) d_{(i)} K_{i}\left(t_{1}, \ldots, t_{i}\right)
$$

and

$$
\int_{0}^{1}[x(t)]^{2} d t \int_{0}^{1} \int_{0}^{1} x\left(t_{1}\right) x\left(t_{2}\right) d_{(2)} K_{2}\left(t_{1}, t_{2}\right)
$$

where the K's are of bounded variation.

Then

$$
\int_{c} F[x(\cdot)] d x=\sum_{k=-\infty}^{\infty} \nu_{k} F\left[x_{k}(\cdot)\right] .
$$


THEOREM 2. Let $F[x(\cdot)]$ be continuous in the Hilbert topology and suppose there exists a positive monotone increasing function $H(u)$ such that

$$
\begin{gathered}
|F[x(\cdot)]| \leqq H\left(\int_{0}^{1} w(t)[x(t)]^{2} d t\right), \\
\int_{c} H\left(\rho+\int_{0}^{1} w(t)[x(t)]^{2} d t\right) d x<\infty
\end{gathered}
$$

for some $\rho>\int_{0}^{1} s w(s) d s / 2\left(\right.$ e.g. $H(u)=\exp (k u)$ with $k<\lambda_{1}$ satisfies (1.5) and (1.6)).

Then

$$
\begin{aligned}
& \int_{c} F[x(\cdot)] d x \\
& =\lim _{n \rightarrow \infty} \sum_{k=-\infty}^{\infty} \nu_{k} \int_{-\infty}^{\infty}(n) \int_{-\infty}^{\infty} e_{n}(\xi) F\left[x_{k}(\cdot)-x_{k}^{n}(\cdot)+\psi_{n}(\xi, \cdot)\right] d \xi_{1} \cdots d \xi_{n} .
\end{aligned}
$$

The proofs for these theorems will be given below but it should first be mentioned that one derivation of the results of the two theorems parallels that given in Vladimirov's paper for his special case $w(t) \equiv 1$. First, constants $\nu_{k}$ and functions $x_{k}(t)$ (which turn out to be as in the notation section) are found to make formula (1.4) valid for all functionals of the forms

$$
K+\sum_{i=1}^{3} x\left(t_{1}\right) \cdots x\left(t_{i}\right)
$$

and

$$
\int_{0}^{1} w(t)[x(t)]^{2} d t x\left(t_{1}\right) x\left(t_{2}\right)
$$

Then Cameron's mixed integration formula is used. That formula, a proof of which (for general complete orthonormal sets of $\alpha$ 's of bounded variation) can be found in [3], states that for any Wiener integrable functional

$$
\begin{aligned}
\int_{c} F & {[x(\cdot)] d x } \\
& =\int_{-\infty}^{\infty}(n) \int_{-\infty}^{\infty} e_{n}(\xi) \int_{c} F\left[x(\cdot)-x^{n}(\cdot)+\psi_{n}(\xi, \cdot)\right] d x d \xi_{1} \cdots d \xi_{n} .
\end{aligned}
$$

Finally, formula (1.10) is applied to the right side of (1.4) and there is shown that in fact (1.7) holds under the conditions stated.

2. The lemmas.

LEMma 2.1. The functions $\left\{\left[\lambda_{i} w(s)\right]^{1 / 2} \beta_{i}(s)\right\}$ are complete orthonormal on $[0,1]$.

LEMMA 2.2.

$$
\int_{0}^{1} w(t)\left[x^{n}(t)\right]^{2} d t \leqq \int_{0}^{1} w(t)[x(t)]^{2} d t
$$

The proof for Lemmas 2.1 and 2.2 will be found in [4]. 
LEMMA 2.3. $\beta_{n}(s)$ satisfies the integral equation

$$
\beta_{n}(s)=\lambda_{n} \int_{0}^{1} w(t) \min (t, s) \beta_{n}(t) d t
$$

Proof. It is easy to show that the Sturm-Liouville problem (1.1) implies that the $\beta$ 's satisfy the conditions

$$
\beta_{n}^{\prime \prime}(s)=-\lambda_{n} w(s) \beta_{n}(s), \quad \beta_{n}(0)=\beta_{n}^{\prime}(1)=0
$$

Thus

$$
\beta_{n}^{\prime}(s)=-\int_{s}^{1} \beta_{n}^{\prime \prime}(t) d t=\lambda_{n} \int_{s}^{1} w(t) \beta_{n}(t) d t
$$

and hence

$$
\begin{aligned}
\beta_{n}(s) & =\int_{0}^{s} \beta_{n}^{\prime}(t) d t=\lambda_{n} \int_{0}^{s} \int_{t}^{1} w(u) \beta_{n}(u) d u d t \\
& =\lambda_{n} \int_{0}^{1} \int_{0}^{1} \rho(t, s) \rho(t, u) w(u) \beta_{n}(u) d u d t \\
& \text { where } \rho(s, t)= \begin{cases}1 & \text { for } 0 \leqq s<t \leqq 1, \\
0 & \text { for } 0 \leqq t \leqq s \leqq 1,\end{cases} \\
= & \lambda_{n} \int_{0}^{1} \int_{0}^{1} \rho(t, s) \rho(t, u) d t w(u) \beta_{n}(u) d u
\end{aligned}
$$

and the proof of the lemma is complete.

LEMMA 2.4 .

$$
\min \left(t_{1}, t_{2}\right)=\sum_{n=1}^{\infty} \beta_{n}\left(t_{1}\right) \beta_{n}\left(t_{2}\right)
$$

Proof. This is just Parseval's equation for the function $\rho\left(s, t_{1}\right) \rho\left(s, t_{2}\right)$ where $\rho(s, t)$ is as in Lemma 2.3, and where the generalized Fourier development is in terms of the $\alpha$ 's (which are a complete orthonormal set of course).

LEMMA 2.5. The $\beta$ 's satisfy the condition

$$
\int_{0}^{1} w(t) \min \left(t, t_{1}\right) \min \left(t, t_{2}\right) d t=\sum_{n=1}^{\infty} \beta_{n}\left(t_{1}\right) \beta_{n}\left(t_{2}\right) / \lambda_{n} .
$$

Proof. This is Parseval's equation for the product

$$
[w(t)]^{1 / 2} \min \left(t, t_{1}\right) \cdot[w(t)]^{1 / 2} \min \left(t, t_{2}\right)
$$


where the development of each of the two factors is given in terms of the set $\left\{\left[\lambda_{i} w(s)\right]^{1 / 2} \beta_{i}(s)\right\}$ which is complete orthonormal according to Lemma 2.1. A verification of this assertion follows. The coefficients in the expansion of $[w(t)]^{1 / 2} \min \left(t, t_{1}\right)$ are given by

$$
\begin{aligned}
& \int_{0}^{1}\left[\lambda_{n} w(t)\right]^{1 / 2} \beta_{n}(t)[w(t)]^{1 / 2} \min \left(t, t_{1}\right) d t \\
& =\left(\lambda_{n}\right)^{1 / 2} \int_{0}^{1} w(t) \min \left(t, t_{1}\right) \beta_{n}(t) d t=\beta_{n}\left(t_{1}\right) / \lambda_{n}^{1 / 2}
\end{aligned}
$$

because of Lemma 2.3. The verification is complete.

LEMMA 2.6. $\sum_{n=1}^{\infty} 1 / \lambda_{n}$ converges.

Proof. If in the Sturm-Liouville problem (1.1) there is made a transformation to new independent and dependent variables $z$ and $y$ by means of the equations

$$
z=\int_{0}^{s}[w(u)]^{1 / 2} d u \text { and } y=\alpha / w^{1 / 4}
$$

there results a differential equation of the type

$$
y^{\prime \prime}+[\lambda-q(z)] y=0
$$

subject to boundary conditions

$$
y(1)=0 \quad \text { and } \quad w^{\prime}(0) y(0) /[4 w(0)]+y^{\prime}(0)=0 .
$$

The theorem [5, p. 155] yields the estimate $\left(\lambda_{n}\right)^{1 / 2} \approx\left[\pi^{2}(n+1 / 2)^{2}+\int_{0}^{1} q(z) d z\right]^{1 / 2}$ to complete the proof of the lemma.

LEMMA 2.7. $\left(\lambda_{n}\right)^{1 / 2} \beta_{n}(t)$ is bounded in $n$ and $t$ for $n=1,2,3, \ldots$ and $t \in[0,1]$.

Proof. For the transformed Sturm-Liouville problem as given in the proof of Lemma 2.6 there follows from [5, p. 146] that the $n$th characteristic function is

$$
y_{n}=c_{n, 1} \sin \left(\lambda_{n}\right)^{1 / 2} z+c_{n, 2} \cos \left(\lambda_{n}\right)^{1 / 2} z+O\left(1 / \lambda_{n}^{1 / 2}\right),
$$

or in terms of the original independent and dependent variables $s$ and $\alpha$

$$
\begin{aligned}
\alpha_{n}(s)= & c_{n, 1}[w(s)]^{1 / 4} \sin \left\{\left(\lambda_{n}\right)^{1 / 2} \int_{0}^{s}[w(u)]^{1 / 2} d u\right\} \\
& +c_{n, 2}[w(s)]^{1 / 4} \cos \left\{\left(\lambda_{n}\right)^{1 / 2} \int_{0}^{s}[w(u)]^{1 / 2} d u\right\} \\
& +O\left(1 / \lambda_{n}^{1 / 2}\right) .
\end{aligned}
$$

In normalizing $\alpha_{n}(s)$ one encounters the integral

$$
\int_{0}^{1}[w(s)]^{1 / 4} \sin \left\{\left(\lambda_{n}\right)^{1 / 2} \int_{0}^{s}[w(u)]^{1 / 2} d u\right\} d s
$$


(and a similar integral involving the cosine function). Since $[w(s)]^{1 / 4}$ does not change sign there follows

$$
\begin{aligned}
& \left|\int_{0}^{1}[w(s)]^{1 / 4} \sin \left\{\left(\lambda_{n}\right)^{1 / 2} \int_{0}^{s}[w(u)]^{1 / 2} d u\right\} d s\right| \\
& \leqq\left|\int_{a}^{b}[w(s)]^{1 / 4} \sin \left\{\left(\lambda_{n}\right)^{1 / 2} \int_{0}^{s}[w(u)]^{1 / 2} d u\right\} d s\right|
\end{aligned}
$$

(where $a$ and $b$ are an appropriate adjacent pair of zeros of

$$
\begin{aligned}
& \left.\left.\leqq\left|\int_{0}^{b}[w(s)]^{1 / 2} \sin \left\{\left(\lambda_{n}\right)^{1 / 2} \int_{0}^{s}[w(u)]^{1 / 2} d u\right\} d s\right| / \min _{0 \leqq s \leqq 1} w(s)^{1 / 4} \int_{0}^{s}[w(u)]^{1 / 2} d u\right\}\right) \\
& =\left.\left|\cos \left\{\left(\lambda_{n}\right)^{1 / 2} \int_{0}^{s}[w(u)]^{1 / 2} d u\right\} /\left[\left(\lambda_{n}\right)^{1 / 2} \min _{0 \leqq s \leqq 1} w(s)^{1 / 4}\right]\right|\right|_{s=a} ^{b} \\
& \leqq 2 /\left[\left(\lambda_{n}\right)^{1 / 2} \min _{0 \leqq s \leqq 1} w(s)^{1 / 4}\right] .
\end{aligned}
$$

In view of the estimate (2.1) there follows easily that if $\alpha_{n}(s): n=1,2,3, \ldots$ is to be normal then

$$
\begin{aligned}
1= & \left(c_{n, 1}^{2}+c_{n, 2}^{2}\right) \int_{0}^{1}[w(s)]^{1 / 2} d s / 2 \\
& +\left(c_{n, 1}^{2}+c_{n, 2}^{2}+c_{n, 1} c_{n, 2}\right) O\left(1 / \lambda_{n}^{1 / 2}\right) \\
& +\left(c_{n, 1}+c_{n, 2}+1\right) O\left(1 / \lambda_{n}\right)
\end{aligned}
$$

and hence $c_{n, 1}$ and $c_{n, 2}$ are bounded in $n$. Thus

$$
\begin{aligned}
\beta_{n}(t)= & \int_{0}^{t} c_{n, 1}[w(s)]^{1 / 4} \sin \left\{\left(\lambda_{n}\right)^{1 / 2} \int_{0}^{s}[w(u)]^{1 / 2} d u\right\} d s \\
& + \text { a similar term }+\int_{0}^{t} O\left(1 / \lambda_{n}^{1 / 2}\right) d s .
\end{aligned}
$$

Since $c_{n, 1}$ and $c_{n, 2}$ have been shown to be bounded, the estimate (2.1) yields at once that

$$
\beta_{n}(t)=O\left(1 / \lambda_{n}^{1 / 2}\right)
$$

to conclude the proof of the lemma.

LEMMA 2.8. The convergence of $\sum_{n=1}^{\infty} \beta_{n}^{2}(t)$ (ensured for all $t \in[0,1]$ by Lemma 2.4) is uniform on $[0,1]$.

Proof. This follows from the fact that if a sequence of monotonically increasing continuous functions converges to a continuous function on a closed interval then the convergence is uniform. Clearly, the convergence of $\sum_{k=1}^{n} \beta_{k}^{2}(t)$ to $t$ is of this type and the proof is complete. 
LEMMA 2.9. The sums

$$
\sum_{n=1}^{\infty}\left[\beta_{n}\left(t_{1}\right)\right]\left[1 /\left(\lambda_{n}\right)^{1 / 2}\right], \quad \sum_{n=1}^{\infty}\left[\beta_{n}\left(t_{1}\right)\right]\left[\beta_{n}\left(t_{2}\right)\right], \quad \sum_{n=1}^{\infty}\left[\left(\lambda_{n}\right)^{1 / 2} \beta_{n}\left(t_{1}\right) \beta_{n}\left(t_{2}\right)\right]\left[\beta_{n}\left(t_{3}\right)\right]
$$

are uniformly convergent on the unit interval, square and cube respectively.

Proof. An application of the Schwartz inequality to the sum of products (in each case the appropriate factoring is indicated by the square brackets) along with the results of Lemmas 2.4 (which implies of course that $\left|\beta_{n}(t)\right| \leqq 1$ ), 2.6, 2.7 and 2.8 completes the proof.

3. Proofs of the theorems. The proof of Theorem 1 now follows.

A standard Wiener integration formula is

$$
\int_{c} x\left(t_{1}\right) x\left(t_{2}\right) d x=\min \left(t_{1}, t_{2}\right) / 2\left({ }^{2}\right) .
$$

Thus from Lemma 2.4 follows

$$
\int_{c} x\left(t_{1}\right) x\left(t_{2}\right) d x=\sum_{n=1}^{\infty} \beta_{n}\left(t_{1}\right) \beta_{n}\left(t_{2}\right) / 2
$$

and this latter sum is easily seen to be $\sum_{n=-\infty}^{\infty} v_{n} x_{n}\left(t_{1}\right) x_{n}\left(t_{2}\right)$. Hence the conclusion of the theorem has been established for functionals of the form $x\left(t_{1}\right) x\left(t_{2}\right)$. It is easy to see that the definitions

$$
\nu_{n}=\nu_{-n} \text { and } x_{n}(t)=-x_{-n}(t)
$$

ensure that the conclusion of the theorem holds for functionals of the form $x\left(t_{1}\right)$ and $x\left(t_{1}\right) x\left(t_{2}\right) x\left(t_{3}\right)$ since the sums converge by Lemma 2.9. Also, the theorem clearly holds, in view of the definition of $\nu_{0}$ for constant functionals. Hence the theorem is established for functionals of the form (1.8). A routine calculation yields that

$$
\begin{aligned}
\int_{c} \int_{0}^{1} w(t)[x(t)]^{2} d t x\left(t_{1}\right) x\left(t_{2}\right) d x= & \int_{0}^{1} t w(t) d t \min \left(t_{1}, t_{2}\right) / 4 \\
& +\int_{0}^{1} w(t) \min \left(t, t_{1}\right) \min \left(t, t_{2}\right) d t / 2 \\
= & \int_{0}^{1} t w(t) d t \sum_{n=1}^{\infty} \beta_{n}\left(t_{1}\right) \beta_{n}\left(t_{2}\right) / 4 \\
& +\sum_{n=1}^{\infty} \beta_{n}\left(t_{1}\right) \beta_{n}\left(t_{2}\right) /\left(2 \lambda_{n}\right)
\end{aligned}
$$

(because of Lemmas 2.4 and 2.5).

Again it is easy to verify that, in view of the definition of $\sigma_{n}$, the last member of the above equation is just what the conclusion of the theorem yields for a functional of the form (1.9). Finally, Fubini's theorem for Lebesgue and Wiener integrals

$\left({ }^{2}\right)$ The difference between this evaluation and that given by Vladimirov is due to the use of a different kernel in the definition of Wiener measure, cf. [2]. 
ensures that the theorem's conclusion holds for functionals of the forms (1.2) and (1.3) because of Lemma 2.9. The proof of Theorem 1 is complete.

Finally there will be given the proof of Theorem 2. Because of (1.10) the right member of (1.7) equals

$$
\lim _{n \rightarrow \infty} \sum_{k=-\infty}^{\infty} \nu_{k} \int_{c} F\left[x_{k}(\cdot)-x_{n}^{k}(\cdot)+x^{n}(\cdot)\right] d x
$$

There will now be noted that, since

it follows that

$$
\int_{0}^{1} \alpha_{i}(s) d \beta_{k}(s)=\int_{0}^{1} \alpha_{i}(s) \alpha_{k}(s) d s=\delta_{k i}
$$

$$
\begin{array}{rlrl}
\beta_{k}^{n}(t) & =\sum_{i=1}^{n} \int_{0}^{1} \alpha_{i}(s) d \beta_{k}(s) \beta_{i}(t) \\
& =\beta_{k}(t) \quad & \text { for } n \geqq k, \\
& =0 & & \text { for } n<k .
\end{array}
$$

Now (3.2) yields that for $n \geqq|k|=K$

$$
\begin{aligned}
\left|F\left[x_{k}(\cdot)-x_{k}^{n}(\cdot)+x^{n}(\cdot)\right]\right| & =\left|F\left[\sigma_{K}\left\{\beta_{K}(\cdot)-\beta_{K}^{n}(\cdot)\right\} / \gamma_{K}^{1 / 2}+x^{n}(\cdot)\right]\right| \\
& \leqq H\left(\int_{0}^{1} w(t)\left[\sigma_{K}\left\{\beta_{K}(t)-\beta_{K}^{n}(t)\right\} / \gamma_{K}^{1 / 2}+x^{n}(t)\right]^{2} d t\right) \\
& =H\left(\int_{0}^{1} w(t)\left[x^{n}(t)\right]^{2} d t\right) \\
& \leqq H\left(\int_{0}^{1} w(t)[x(t)]^{2} d t\right) \quad \text { (because of Lemma 2.2) } \\
& \leqq H\left(\rho+\int_{0}^{1} w(t)[x(t)]^{2} d t\right)
\end{aligned}
$$

while for any $n$ and for sufficiently large $|k|=K>n$

$$
\begin{aligned}
&\left|F\left[x_{k}(\cdot)-x_{k}^{n}(\cdot)+x^{n}(\cdot)\right]\right|=\left|F\left[\sigma_{K}\left\{\beta_{K}(\cdot)-\beta_{K}^{n}(\cdot)\right\} / \gamma_{K}^{1 / 2}+x^{n}(\cdot)\right]\right| \\
& \leqq H\left(\int_{0}^{1} w(t)\left[\sigma_{K} \beta_{K}(t) / \gamma_{K}^{1 / 2}+x^{n}(t)\right]^{2} d t\right) \\
&=H\left(\int_{0}^{1} w(t)\left[\sigma_{K}^{2} \beta_{K}^{2}(t) / \gamma_{K}+\sum_{i=1}^{n} c_{i}^{2} \beta_{i}^{2}(t)\right] d t\right) \\
&\left.=H\left(\sigma_{K}^{2} /\left[\lambda_{K} \gamma_{K}\right]+\sum_{i=1}^{n} c_{i}^{2} / \lambda_{i}\right) \quad \text { (because } n<K\right) \\
& \leqq H\left(\sigma_{K}^{2} /\left[\lambda_{K} \gamma_{K}\right]+\int_{0}^{1} w(t)[x(t)]^{2} d t\right) \\
& \leqq H\left(\rho+\int_{0}^{1} w(t)[x(t)]^{2} d t\right) \quad \text { (by Lemma 2.2) } \\
&\text { (because } \left.\sigma_{K}^{2} /\left[\lambda_{K} \gamma_{K}\right]=\int_{0}^{1} s w(s) d s / 2-1 / \lambda_{K} \text { and } \lim _{k \rightarrow \infty} 1 / \lambda_{k}=0\right) .
\end{aligned}
$$


From (3.1) there follows that for arbitrary $N>0$ the limitand of the right member of (1.7) equals (where $|k|=K$ )

$$
\begin{aligned}
\sum_{k=-N}^{N} \nu_{k} \int_{c} F\left[\sigma_{K}\left\{\beta_{K}(\cdot)-\beta_{K}^{n}(\cdot)\right\} / \gamma_{K}^{1 / 2}\right. & \left.+x^{n}(\cdot)\right] d x \\
& +\sum_{|k|>N} \nu_{k} \int_{c} F\left[\sigma_{K}\left\{\beta_{K}^{n}(\cdot)-\beta_{K}^{2}(\cdot)\right\} / \gamma_{K}^{1 / 2}+x^{n}(\cdot)\right] d x .
\end{aligned}
$$

Since

$$
\left|\int_{c} F\left[\sigma_{K}\left\{\beta_{K}(\cdot)-\beta_{K}^{n}(\cdot)\right\} / \gamma_{K}^{1 / 2}+x^{n}(\cdot)\right] d x\right| \leqq \int_{c} H\left(\rho+\int_{0}^{1} w(t)[x(t)]^{2} d t\right) d x
$$

for all $n$ and sufficiently large $K>N$, and since $\sum_{k=-\infty}^{\infty} \nu_{k}$ converges (to 1 ) it follows that $N$ can be chosen so large that the second member of the limitand is arbitrarily small and so that also $\sum_{k=-N}^{N} \nu_{k}$ is arbitrarily close to 1 . Let $N$ be chosen even larger, if need be, so that for $n=N, \int_{c} F\left[\sigma_{K}\left\{\beta_{K}(\cdot)-\beta_{K}^{n}(\cdot)\right\} / \gamma_{K}^{1 / 2}+x^{n}(\cdot)\right] d x\left(=\int_{c} F\left[x^{n}(\cdot)\right] d x\right.$ for $K \leqq N$, cf. (3.2)) is nearly equal to $\int_{c} F[x(\cdot)] d x$ for $K \leqq N$. Thus the first member of the limitand can be made arbitrarily close to $\int_{c} F[x(\cdot)] d x$ and the second member arbitrarily small to complete the proof of (1.7) under the stated conditions. Finally that there exists an $H(u)$ of the type required in theorem, viz. $H(u)=$ $\exp (k u)$ with $k<\lambda_{1}$ is proven in [4].

\section{REFERENCES}

1. R. H. Cameron, $A$ "Simpson's Rule" for the numerical evaluation of Wiener's integrals in function space, Duke Math. J. 18 (1951), 111-130.

2. Phys. (2) 39 (1960), 128.

3. Henry C. Finlayson, New approximations for Wiener integrals, with error estimates, Canad. J. Math. 19 (1967), 58-105.

4. - An approximation method for Wiener integrals of certain unbounded functionals, Proc. Amer. Math. Soc. 18 (1967), 798-803.

5. Harry Hochstadt, Differential equations, a modern approach, Stechert-Hafner, New York, 1964.

6. V. S. Vladimirov, The approximate evaluation of Wiener integrals, Uspehi Mat. Nauk 15 (1960), no. 4 (94), 129-135. (Russian)

\section{UNIVERSITY OF MANITOBA,}

WinNIPEg, MaNitoba, CANAdA 